PROCEEDINGS OF THE

AMERICAN MATHEMATICAL SOCIETY

Volume 29, Number 1, June 1971

\title{
A LIMITATION THEOREM FOR ABSOLUTE SUMMABILITY
}

\author{
GODFREY L. ISAACS
}

Abstract. Let $A(u)$ be of bounded variation over every finite interval of the nonnegative real axis, and let $\int_{0}^{w} e^{-u s} d A(u)$ be summable $|C, k|$ for a given integer $k \geqq 0$ and a given $s$ whose real part is negative. Then it is known that the function $R(k, w)=(1 / \Gamma(k+1))$ $\cdot \int_{w}^{\infty}(u-w)^{k} d A(u)$ (which certainly exists in the $|C, k|$ sense by a well-known summability-factor theorem) satisfies $e^{-w s} w^{-k} R(k, w)$ $=o(1)|C, 0|(w \rightarrow \infty)$. In this paper we extend the above result by showing that if the hypotheses are satisfied with $k$ fractional, then $e^{-w s} w^{-k} R(k+\delta, w)=o(1)|C, 0|$ for each $\delta>0$ and that this is best possible in the sense that $\delta$ may not be replaced by 0 .

1. Let $A(w)$ be of bounded variation over every finite interval of the nonnegative real axis. We write

$$
F(a ; x)=\int_{a}^{x} f(u) d A(u)=L+o(1) \quad(C, k)
$$

(read: $F(a ; x)$ is summable $(C, k)$ to the limit $L$, or $\int_{a}^{\infty} f(u) d A(u)$ exists in the $(C, k)$ sense and equals $L)$ if

$$
\Gamma(k+1) x^{-k} F_{k}(a ; x)=x^{-k} \int_{a}^{x}(x-u)^{k} f(u) d A(u) \rightarrow L
$$

as $x \rightarrow \infty$. (Stieltjes integrals are to be taken in the Riemann sense.) If in addition $x^{-k} F_{k}(a ; x)$ is of bounded variation over $[a, \infty)$ we shall write $|C, k|$ instead of $(C, k)$ in the notations above.

This paper is concerned with the $(C, k)$ and $|C, k|$ summability of

$$
C(x) \quad(=C(0 ; x))=\int_{0}^{x} e^{-u s} d A(u)
$$

and of

$$
R\left(k^{\prime}, w ; x\right)=1 / \Gamma\left(k^{\prime}+1\right) \int_{w}^{x}(u-w)^{k^{\prime}} d A(u)
$$

Presented to the Society, April 28, 1969; received by the editors October 7, 1970.

AMS 1970 subject classifications. Primary 40A10, 40F05, 40G05; Secondary 40D05, 40D15. $|C, k|$.

Key words and phrases. Laplace-Stieltjes integral, Cesàro summability, summable Copyright (C) 1971, American Mathematical Society 
We shall write

$$
R\left(k^{\prime}, w\right)=\left(1 / \Gamma\left(k^{\prime}+1\right)\right) \int_{w}^{\infty}(u-w)^{k^{\prime}} d A(u)
$$

so that $R\left(k^{\prime}, w\right)$ exists in the $(C, k)$ sense iff (3) is summable $(C, k)$. In virtue of $[1$, p. 300], if (2) is summable $(C, k)$ (or $|C, k|$ ) for some $k \geqq 0, \operatorname{Re}(s)<0$, then $R\left(k^{\prime}, w\right)$ exists in the $(C, k)$ (or $|C, k|$ ) sense for each $w \geqq 0, k^{\prime} \geqq 0$. We have now :

Theorem A [5, pp. 412-413]. If $k=0,1,2, \cdots$, and (2) is summable $|C, k|$, where $\operatorname{Re}(s)=\sigma<0$, then

$$
e^{-w s} w^{-k} R(k, w)=o(1) \quad|C, 0| .
$$

The last phrase will mean that the function on the left, $g(w)$, say, tends to 0 as $w \rightarrow \infty$ and is of bounded variation over $[1, \infty)$, i.e.,

$$
\int_{1}^{w} d g(u)=-g(1)+o(1) \quad|C, 0| .
$$

We state now, writing $[k]$ for the largest integer $\leqq k$, and $\langle k\rangle$ for $k-[k]$ :

THEOREM $\mathrm{A}^{\prime}$. If $k$ is positive and fractional, and if (2) is summable $|C, k|$ for some such that $\sigma<0$, then

$$
e^{-w s} w^{-k} R(k, w)=B(w)+(-1)^{[k]+1} w^{-k} T(w),
$$

where $B(w)=o(1)|C, 0|$ and

$$
T(w)=1 / \Gamma(\langle k\rangle) \int_{w}^{w+1}(u-w)^{\langle k\rangle-1} C_{[k]}(u) d u,
$$

$C(u)$ being given by (2).

ThEOREM A". Under the hypotheses of Theorem $\mathrm{A}^{\prime}$,

$$
e^{-w s} w^{-k} R(k+\delta, w)=o(1) \quad|C, 0| \quad \text { for each } \delta>0 .
$$

THEOREM $\mathrm{A}^{\prime \prime \prime}$. Under the hypotheses of Theorem $\mathrm{A}^{\prime}, e^{-w s} w^{-k} R(k, w)$ is not necessarily bounded, even with $e^{-w s}$ replaced by $e^{-w X}$ with $X$ as large as we please.

Theorem $\mathrm{A}^{\prime \prime}$ is the extension of Theorem A to the case $k$ fractional, and Theorem $A^{\prime \prime \prime}$ shows that Theorem $A^{\prime \prime}$ is best possible in the sense that $\delta$ may not be replaced by 0 .

2. We shall prove the following slight generalization of Theorem $\mathrm{A}^{\prime}$ : 
TheOREM $\mathrm{A}^{\prime *}$. If $C(w)$ is summable $|C, k-\delta|$, where $k$ is positive and fractional, and $\sigma<0,0 \leqq \delta<\langle k\rangle$, then

$$
e^{-w s} w^{\delta-k} R(k, w)=B^{(\delta)}(w)+(-1)^{[k]+1} w^{\delta-k} T(w)
$$

where $B^{(\delta)}(w)=o(1)|C, 0|$ and $T(w)$ is given by (5).

By [6], slightly modified, the $(C)$ versions of Theorems $\mathrm{A}^{\prime *}$ and $\mathrm{A}^{\prime \prime}$ (obtained by replacing $|C, \ldots|$ by $(C, \cdots)$ ) hold. Thus it is sufficient to prove $A^{\prime *}$ and $A^{\prime \prime}$ with ' $=o(1)$ ' replaced by 'is summable'. We shall use (see $[6,(25)-(31)])$ :

LemMa 1. If for a given $k \geqq 0$ and $\sigma<0, C(w)$ is summable $(C,[k]+1)$, then $R(k, w)$ exists in the $(C,[k]+1)$ sense and

where

$$
R(k, w)=\sum_{v=0}^{[k]+1} b_{v} Q(k, v, w)
$$

$$
Q(k, v, w)=\int_{w}^{\infty} C_{[k]}(u)(u-w)^{k-v} e^{u s} d u
$$

the integrals being convergent, and the b's being constants, with

$$
b_{[k]+1}=(-1)^{[k]+1} / \Gamma(\langle k\rangle) .
$$

Theorems $\mathrm{A}^{\prime *}$ and $\mathrm{A}^{\prime \prime}$ will be deduced from

Theorem A**. Under the hypotheses of Theorem $\mathrm{A}^{\prime *}, e^{-w s} w^{\delta-k}$ - $Q(k, v, w)$ is summable $|C, 0|$ if either (i) $0<\delta<\langle k\rangle, v \leqq[k]+1$, or (ii) $\delta=0, v \leqq[k]$.

We shall require

LEMMA 2. Let $w \geqq 1,-\infty \leqq a<b \leqq \infty$, and $a<u<b$. If

then

$$
F(w)=\int_{a}^{b} g(w, u) f(u) d u \text { and } \int_{1}^{\infty}\left|d_{w} g(w, u)\right| \leqq g(u),
$$

$$
\int_{1}^{\infty}|d F(u)| \leqq \int_{a}^{b} g(u)|f(u)| d u,
$$

the integrals over $(a, b)$ being supposed existent in the Lebesgue sense.

PROOF. If $w_{0}=1<w_{1}<\cdots<w_{m}$ we have

$$
\sum_{n=1}^{m}\left|F\left(w_{n}\right)-F\left(w_{n-1}\right)\right| \leqq \int_{a}^{b}|f(u)| d u \sum_{n=1}^{m}\left|g\left(w_{n}, u\right)-g\left(w_{n-1}, u\right)\right|
$$

and the sum on the right is $\leqq g(u)$ by hypothesis. 
Proof of Theorem A**. We write

$$
\begin{aligned}
p(t) & =t^{\delta-k} C_{k-\delta}(t) & & (t>0), \\
& =0 & & (t=0) .
\end{aligned}
$$

Then $p(t)$ is of bounded variation over $[0, \infty)$. Let

$$
D(u, w)=C_{[k]+1}(u)-C_{[k]+1}(w) .
$$

Then integrating by parts in (6) and using $C_{[k]+1}(u)=O\left(u^{[k]+1}\right)$, we have $w^{\delta-k} e^{-w s} Q(k, v, w)=(v-k) I_{v}-s I_{v-1}$ where

$$
I_{v}=w^{\delta-k} \int_{w}^{\infty}(u-w)^{k-v-1} e^{(u-w) s} D(u, w) d u .
$$

Now $\Gamma(\delta-\langle k\rangle+1) D(u, w)$ can be expressed as

$$
\int_{0}^{u}(u-t)^{\delta-\langle k\rangle} C_{k-\delta}(t) d t-\int_{0}^{w}(w-t)^{\delta-\langle k\rangle} C_{k-\delta}(t) d t .
$$

We write the first integral as the sum of integrals over $[w, u]$ and $[0, w]$ and then combine the second of these with the second integral in (11), thus obtaining $X+Y$, say. We replace $C_{k-\delta}(t)$ by $t^{k-\delta} p(t)$ in each of these, and then put $t=w+(u-w) y$ in $X$ and $t=w-x$ in $Y$. Inserting the resultant expression in (10) and putting $u=w+z$, we obtain for $\Gamma(\delta-\langle k\rangle+1) I_{0}$ :

$$
\int_{0}^{\infty} z^{\delta+[k]-v} e^{z s} d z \int_{0}^{1}(1-y)^{\delta-\langle k\rangle} r(z, y, w) p(w+z y) d y
$$

$$
\begin{aligned}
& -\int_{0}^{\infty} z^{k-v-1} e^{z s} d z \int_{0}^{w}\left\{x^{\delta-\langle k\rangle}-(x+z)^{\delta-\langle k\rangle}\right\}(1-x / w)^{k-\delta} p(w-x) d x \\
& =L(w)-M(w),
\end{aligned}
$$

say, where $r(z, y, w)=(1+z y / w)^{k-\delta}$. Since $r$ decreases as $w$ increases,

$$
\begin{aligned}
& \int_{1}^{\infty}\left|d_{w}(r p(w+z y))\right| \leqq \int_{1}^{\infty}|p(\partial r / \partial w)| d w \\
&+\int_{1}^{\infty} r\left|d_{w} p(w+z y)\right| \leqq c(1+z)^{k-\delta}
\end{aligned}
$$

say, where $c$ is independent of $z$ and $y$. Hence by Lemma 2,

$$
\int_{1}^{\infty}|d L(w)| \leqq c \int_{0}^{\infty}(1+z)^{k-\delta} z^{\delta+[k]-v} e^{z \sigma} d z \int_{0}^{1}(1-y)^{\delta-\langle k\rangle} d y,
$$


which is finite in either case (i) or case (ii). Next, let

Then

$$
\begin{aligned}
q(w, x) & =(1-x / w)^{k-\delta} p(w-x) & & (0 \leqq x<w), \\
& =0 & & (x \geqq w) .
\end{aligned}
$$

$$
\int_{1}^{\infty}\left|d_{w} q(w, x)\right| \leqq \int_{x}^{\infty}\left|d_{w}\left((1-x / w)^{k-\delta} p(w-x)\right)\right| \leqq c^{\prime},
$$

where $c^{\prime}$ is independent of $x$, by an argument similar to (13). Hence by Lemma 2 ,

$$
\int_{1}^{\infty}|d M(w)| \leqq c^{\prime} \int_{0}^{\infty} z^{k-v-1} e^{z \sigma} d z \int_{0}^{\infty}\left\{x^{\delta-\langle k\rangle}-(x+z)^{\delta-\langle k\rangle}\right\} d x
$$

which is finite in either case (i) or case (ii). Since, finally, each of these cases is satisfied by $v-1$ if it is satisfied by $v$, the proof of Theorem A** is complete.

Proof of Theorem $\mathrm{A}^{\prime \prime}$. Put $k-\delta=k^{\prime}$ in Theorem $\mathrm{A}^{* *}$, case (i). Then by Lemma 1 the function $S^{(\delta)}(w)=e^{-w s} w^{-k^{\prime}} R\left(k^{\prime}+\delta, w\right)$ is of bounded variation over $[1, \infty)$ for each sufficiently small $\delta>0$. Now by $[6$, Lemma 2$]$ we have, for $p>0$,

$$
e^{w s} w^{k^{\prime}} S^{(\delta+p)}(w)=1 / \Gamma(p) \int_{w}^{\infty}(u-w)^{p-1} e^{u s} u^{k^{\prime}} S^{(\delta)}(u) d u
$$

The substitution $u=w+x$ followed by an application of (our) Lemma 2 and an argument like that of (13) gives $S^{(\delta+p)}(w)$ of bounded variation over $[1, \infty)$. This completes the proof.

Proof of Theorem $\mathrm{A}^{\prime *}$. By either case (i) or case (ii) of Theorem $A^{* *}$, together with Lemma 1 , we have for $0 \leqq \delta<\langle k\rangle$,

$$
w^{\delta-k} e^{-w s} R(k, w)=H(w)+w^{\delta-k} e^{-w s} b_{[k]+1} Q(k,[k]+1, w),
$$

where $H(w)$ is of bounded variation over $[1, \infty)$. We write, by (9),

$$
\begin{aligned}
Q(k,[k]+1, w)= & \left(\int_{w}^{w+1}+\int_{w+1}^{\infty}\right)(u-w)^{\langle k\rangle-1} e^{u s}(\partial D / \partial u) d u \\
= & J+K, \\
J= & e^{w s} \int_{w}^{w+1}(u-w)^{\langle k\rangle-1} C_{[k]}(u) d u \\
& +\int_{w}^{w+1}(u-w)^{\langle k\rangle-1}\left(e^{u s}-e^{w s}\right) \frac{\partial D}{\partial u} d u \\
= & J_{1}+J_{2} .
\end{aligned}
$$


Integrations by parts of $K$ and $J_{2}$, followed by arguments along the lines of (11)-(14), show that $e^{-w s} w^{\delta-k}\left(K+J_{2}\right)$ is of bounded variation over $[1, \infty)$. By $(7)$ this completes the proof.

Proof of TheOrem $A^{\prime \prime \prime}$. We shall use

Lemma 3. Suppose that $k$ is positive and fractional and that $y_{n}$ $(n=1,2, \cdots)$ is a given sequence of positive numbers tending monotonically to $\infty$. Then there exists a function $C(u)$ such that

(a) $C(u)$ is absolutely continuous over every finite interval of the nonnegative real axis, and $C(0)=0$;

(b) $C(u)=o(1)|C, k|$;

but such that the function $T(w)$ given by (5) satisfies $-T(2 n) \geqq c^{\prime} y_{n}(n$ $=1,2, \cdots)\left(c^{\prime}\right.$ a positive constant $)$.

Proof. Let $b, c$ satisfy $0<c-b<2$. We define (compare [3, p. 286]) a function $g_{b, c}(x)$ with domain $b \leqq x \leqq c$, such that it is symmetric about $x=(b+c) / 2$ and

$$
g_{b, c}(x)=\left(1-E^{[k]+2}\right)^{[k]+2} \quad(b \leqq x \leqq(b+c) / 2),
$$

where $E=(b+c-2 x) /(c-b)$. By induction on $r, g_{b, c}^{(r)}(x)$ has a factor $\left(1-E^{[k]+2}\right)^{[k]+2-r} E^{[k]+2-r}$ for $b \leqq x \leqq(b+c) / 2 \quad(r=1,2, \cdots,[k]+1)$, and thus $g_{b, c}^{(r)}(x)$ is 0 at $x=b, c,(b+c) / 2$. The latter (with $x=b, c$ ) is clearly true also for $r=0$. For $r=[k]+2$ the function exists and is bounded in $b<x<(b+c) / 2$ and in $(b+c) / 2<x<c$. Further,

$$
\int_{b}^{(b+c) / 2}\left|g_{b, c}^{\prime}(x)\right| d x=\int_{(b+c) / 2}^{c}\left|g_{b, c}^{\prime}(x)\right| d x=1 .
$$

We now write $h_{n}=\frac{1}{2} e^{-n y_{n}}$, and define $G(u)$ as follows: for $0 \leqq u \leqq 1$, $G(u)=0$; and for $u \geqq 1$ we have, taking $n=1,2, \cdots$,

$$
\begin{aligned}
G(u) & =0 & & (2 n \leqq u<2 n+1), \\
& =1 / n & & \left(2 n-1+h_{n} \leqq u<2 n-h_{n}\right), \\
& =(1 / n) g_{2 n-1,2 n-1+2 h_{n}}(u) & & \left(2 n-1 \leqq u<2 n-1+h_{n}\right), \\
& =(1 / n) g_{2 n-2 h_{n}, 2 n}(u) & & \left(2 n-h_{n} \leqq u<2 n\right) .
\end{aligned}
$$

Then $0 \leqq G(u) \leqq 1$ for all $u>0$. We see that $G$ has a $[k]+1$ th derivative everywhere, and a $[k]+2$ th derivative almost everywhere, which is bounded on every finite interval. Hence we may choose $C(u)$ such that $C_{k}(u)=G(u), C(0)=0$, and $C(u)$ is absolutely continuous over 
every finite interval of the nonnegative real axis. Now by differentiating on the left side we have

$$
\int_{1}^{\infty}\left|\frac{d}{d u}\left(u^{-k} C_{k}(u)\right)\right| d u \leqq 1+\sum_{n=1}^{\infty}\left(\int_{2 n-1}^{2 n}+\int_{2 n}^{2 n+1}\right) u^{-k}\left|C_{k^{\prime}}(u)\right| d u
$$

The second integral on the right is 0 ; and by (17) and (16) the first is $\leqq(2 n-1)^{-k} n^{-1}(1+1)$, so that the sum is finite. Hence (15) is established. We now write, by (5),

$$
\begin{aligned}
-\Gamma(\langle k\rangle) \Gamma(1 & -\langle k\rangle) T(2 n) \\
& =-\int_{2 n}^{2 n+1}(u-2 n)^{\langle k\rangle-1} d u \int_{0}^{u}(u-t)^{-\langle k\rangle} C_{k^{\prime}}(t) d t .
\end{aligned}
$$

We call this expression I. Replacing $u$ by $2 n$ in the inner integral (since $C_{k^{\prime}}(t)=0$ for $\left.2 n \leqq u \leqq 2 n+1\right)$, then integrating the latter by parts, and thereafter using the fact that the resulting integral is decreased by replacing its limits by $2 n-1+h_{n}$ and $2 n-h_{n}$, we obtain, after an inversion,

$$
\mathrm{I} \geqq\langle k\rangle \int_{p}^{q} C_{k}(t) d t \int_{2 n}^{2 n+1}(u-2 n)^{\langle k\rangle-1}(u-t)^{-\langle k\rangle-1} d u,
$$

where $p=2 n-1+h_{n}, q=2 n-h_{n}$. Writing $u-t$ as

$$
\left(1-\frac{2 n+1-u}{2 n+1-t}\right)(2 n+1-t)
$$

expanding the $(-\langle k\rangle-1)$ th power of the first factor in a binomial series and then integrating term by term, we see that the last inner integral is $\langle k\rangle^{-1}(2 n+1-t)^{-\langle k\rangle}(2 n-t)^{-1}$. Hence by (17),

$$
\mathrm{I} \geqq n^{-1} \int_{p}^{q}(2 n+1-t)^{-\langle k\rangle}(2 n-t)^{-1} d t \geqq n^{-1} 2^{-\langle k\rangle} \log \frac{2 n-p}{2 n-q} .
$$

The definitions of $p, q, h_{n}$, now give $\mathrm{I} \geqq 2^{-\langle k\rangle} y_{n}$, which completes the proof.

Proof of Theorem $\mathrm{A}^{\prime \prime \prime}$. For the given $s$, let $y_{n}=e^{e^{2 n}} e^{-2 n \sigma}$. Let $C(u)$ satisfy the conditions of Lemma 3 , with this $y_{n}$.

Choosing $A(u)=\int_{0}^{u} e^{t_{s}} d C(t)$, we see that by Theorem $\mathrm{A}^{\prime}$,

$$
R(k, 2 n)=e^{2 n s}(2 n)^{k} B(2 n)+(-1)^{[k]+1} e^{2 n s} T(2 n),
$$

where the term involving $B(2 n)$ tends to 0 as $n \rightarrow \infty$. But then 
$|R(k, 2 n)| \geqq c^{\prime \prime} e^{2 n}$ for all $n$ large enough, where $c^{\prime \prime}$ is a positive constant. This completes the proof.

In conclusion, I wish to thank Professor D. Borwein for his comments and for Lemma 2 and Theorem $A^{* *}$, which greatly reduced the complexity of my original proofs; also Professor W. H. J. Fuchs for his valuable suggestions.

\section{BiBLIOGRAPHY}

1. L. S. Bosanquet, The summability of Laplace-Stieltjes integrals, Proc. London Math. Soc. (3) 3 (1953), 267-304. MR 15, 307.

2. - The summability of Laplace-Stieltjes integrals. II, Proc. London Math. Soc. (3) 11 (1961), 654-690. MR 25 \#5352.

3. J. Cossar, A note on Cesdro summability of infinite integrals, J. London Math. Soc. 25 (1950), 284-289. MR 12, 253.

4. G. L. Isaacs, On a limitation theorem for Laplace integrals, J. London Math. Soc. 28 (1953), 329-335. MR 15, 307.

5. - An extension of a limitation theorem of M. Riesz, J. London Math. Soc. 33 (1958), 406-418. MR 21 \#270.

6. - On the summability-abscissae of Laplace integrals, Proc. London Math. Soc. (3) 10 (1960), 461-479. MR 22 \#9815.

Herbert H. Lehman College, City University of New York, Bronx, New YORK 10468 